\title{
Solution of Axisymmetric Inhomogeneous Problems with the Markov Chain Monte Carlo
}

\author{
Adebowale E. Shadare ${ }^{1}$, Matthew N. O. Sadiku ${ }^{2}$ and Sarhan M. Musa ${ }^{3}$ \\ ${ }^{1,2}$ Department of Electrical/Computer Engineering, Prairie View A\&M University, Texas, United States \\ ${ }^{3}$ Department of Engineering Technology, Prairie View A\&M University, Texas, United States \\ *corresponding author, E-mail: shadareadebowale@yahoo.com
}

\begin{abstract}
With increasing complexity of EM problems, $1 \mathrm{D}$ and 2D axisymmetric approximations in $\rho, \boldsymbol{z}$ plane are sometimes necessary to quickly solve difficult symmetric problems using limited data storage and within shortest possible time. Inhomogeneous EM problems frequently occur in cases where two or more dielectric media, separated by an interface, exist and could pose challenges in complex EM problems. Simple, fast and efficient numerical techniques are constantly desired. This paper presents the application of simple and efficient Markov Chain Monte Carlo (MCMC) to EM inhomogeneous axisymmetric Laplace's equations. Two cases are considered based on constant and mixed boundary potentials and MCMC solutions are found to be in close agreement with the finite difference solutions.
\end{abstract}

\section{Introduction}

Homogeneous and inhomogeneous Laplace's equations with Dirichlet boundary conditions in Cartesian coordinates have been extensively studied using the MCMC method [1]-[6]. However, with increasing complexity of electromagnetic (EM) problems, 1D and 2D axisymmetric approximations in $\rho, \mathbf{z}$ plane are sometimes necessary to quickly solve difficult symmetric problems using limited data storage resources and within shortest possible time. Inhomogeneous EM problems frequently occur in cases where two or more different dielectric media separated by an interface exist and could pose challenges in complex EM problems. The knowledge of electric field and potential distributions in electronic and high voltage components, with multidielectric interfaces, is often required to ensure accurate prediction of components practical performance [7]. So simple numerical techniques are constantly sought and desired especially in solution of problems with discrete inhomogeneities.

Ever since the Monte Carlo method was first introduced by J. von Neumann and S. M. Ulam, various kinds of Monte Carlo methods such as fixed random walk, floating random walk and Exodus methods have evolved. These Monte Carlo methods have generously been applied in broad areas of engineering including semiconductors, heat conduction, power engineering, electrostatics, waveguide analysis, and antennas [8]-[15]. Classical Monte Carlo methods like the fixed random walk, floating random walk, Exodus method have all been used successfully as numerical techniques for field computation in spite of their major limitation that they only allow single point calculations. Later, the shrinking boundary and inscribed figure methods were proposed for whole-field calculation but they still offered no significant advantage over the conventional Monte Carlo techniques [16]-[17]. Andrey Markov proposed the Markov Chains method that proved to be more efficient than shrinking boundary and inscribed figure methods for whole field computations [18]-[19]. The method is simple, accurate and robust in terms of implementation. The Markov Chain Monte Carlo (MCMC) method involves no use of random number generator and thus not subject to randomness and the approach is potentially accurate [19]. Hence, the MCMC method is generally preferred for whole field computation.

Some reported works in the literature on axisymmetric electrostatic problems with the Monte Carlo methods are presented in [20]-[21]. Solutions of axisymmetric problems by other methods are presented in [22]-[33]. However, in this paper, we propose the application of simple and efficient MCMC method to the solution of axisymmetric inhomogeneous Laplace's equations. To the best of the authors' knowledge, solution of axisymmetric inhomogeneous Laplace's equations with the MCMC is rare or not yet reported in the literature.

\section{Inhomogeneous Media}

Media Inhomogeneity occurs when two or more media have dielectric permittivity difference. The system is anisotropic across the two media but isotropic within each medium. Such a system is represented in the Figure 1.

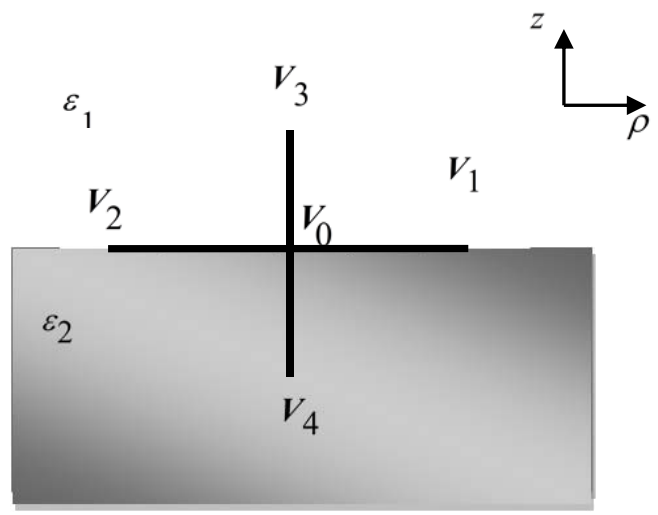

Figure 1. Interface between media of dielectric permittivity

$$
\varepsilon_{1} \text { and } \varepsilon_{2} \text { [34]. }
$$


The Figure 1 shows the interface between two media of dielectric permittivities $\varepsilon_{1}$ and $\varepsilon_{2}$.

For the $Z=$ constant interface, the $D_{1 n}=D_{2 n}$ or $\varepsilon_{1} \frac{\partial V_{1}}{\partial z}=\varepsilon_{2} \frac{\partial V_{2}}{\partial z}$

The transition probabilities at the interface are determined as [21], [34]:

$$
\begin{aligned}
& p_{z+}=\frac{\varepsilon_{1}}{2\left(\varepsilon_{1}+\varepsilon_{2}\right)} \\
& p_{z-}=\frac{\varepsilon_{2}}{2\left(\varepsilon_{1}+\varepsilon_{2}\right)} \\
& p_{\rho+}=p_{\rho-}=\frac{1}{4}
\end{aligned}
$$

For the $\rho=$ constant interface, the $D_{1 n}=D_{2 n}$ or

$\frac{\varepsilon_{1}}{\rho} \frac{\partial V_{1}}{\partial \rho}=\frac{\varepsilon_{2}}{\rho} \frac{\partial V_{2}}{\partial \rho}$, leading to transition probabilities [21]:

$p_{\rho+}=\frac{\varepsilon_{1}}{2\left(\varepsilon_{1}+\varepsilon_{2}\right)}\left(1+\frac{\Delta}{2 \rho}\right)$

$p_{\rho-}=\frac{\varepsilon_{2}}{2\left(\varepsilon_{1}+\varepsilon_{2}\right)}\left(1-\frac{\Delta}{2 \rho}\right)$

However, $p_{z_{+}}$and $p_{z_{-}}$remain the same as in the equations (3).

\section{Inhomogeneous Boundary Conditions}

The inhomogeneous boundary conditions are presented in this section. In general, we have [34]-[35],

- $\quad$ Dirichlet boundary condition:

$$
V(r)=p(r), \boldsymbol{r} \text { on } \boldsymbol{S}
$$

- Neumann boundary condition:

$$
\frac{\partial V(r)}{\partial n}=q(r), \boldsymbol{r} \text { on } \boldsymbol{S}
$$

The normal directive of $\boldsymbol{V}$ vanishes on $\boldsymbol{S}$.

- $\quad$ Mixed boundary condition:

$$
\frac{\partial V(r)}{\partial n}+h(r) V(r)=w(r), \boldsymbol{r} \text { on } \boldsymbol{S}
$$

where $p(r), q(r)$, and $w(r)$ are explicitly known functions on the boundary $S$.

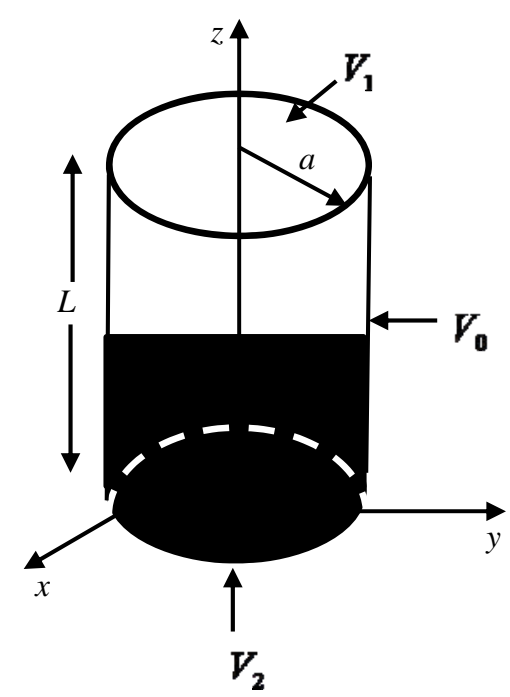

Figure 2. Inhomogeneous Cylindrical Geometry.

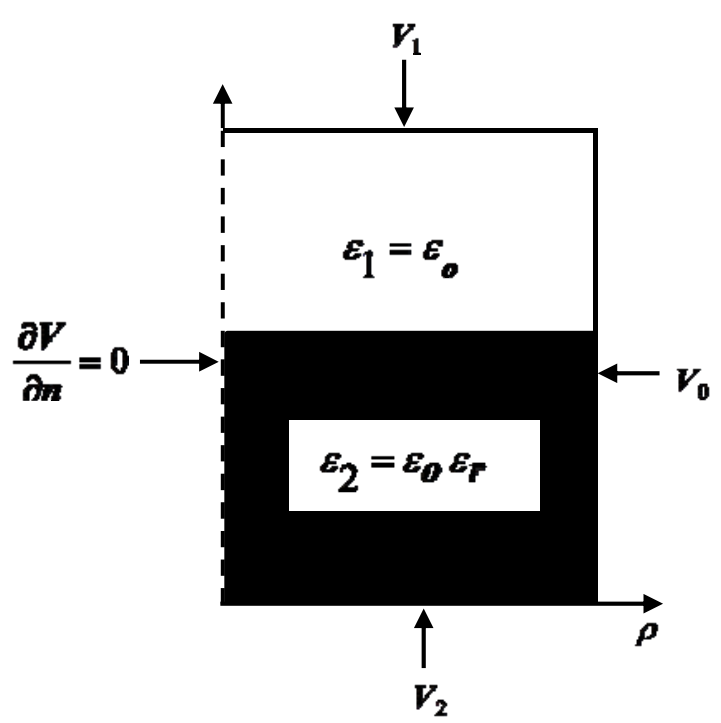

Figure 3. Inhomogeneous Axisymmetric Solution Region

\section{Inhomogeneous Axisymmetric Problem Formulation}

Suppose an inhomogeneous cylindrical coordinate system is as shown in the Figure 2 and the corresponding axisymmetric approximation in $\rho, \mathbf{z}$ plane is as shown in the Figure 3. In the Figure 2, the boundary conditions and source terms $V_{0}, V_{1}$ and $V_{2}$ are symmetric around the cylinder's axis and thus, axisymmetric approximation can be deployed. The approximation reduces the complexity of the system, saves time and reduces possibility of errors. 
It is apparent that the cylinder in the Figure 2 presents inhomogeneous problems at the interface of the two media. The medium 1 consists of air while medium 2 consists of a certain dielectric material. The MCMC procedures for inhomogeneous problems are essentially the same as for the homogeneous problems. However, at the interface between the two media of dielectric permittivity $\varepsilon_{1}$ and $\varepsilon_{2}$, the transition probabilities are determined differently.

In the axisymmetric solution region $\mathbf{R}$, we have

$\nabla^{2} V=\frac{\partial^{2} V}{\partial \rho^{2}}+\frac{1}{\rho} \frac{\partial V}{\partial \rho}+\frac{\partial^{2} V}{\partial z^{2}}=0$

with the corresponding finite difference approximation given in the equation (24), for square grid, $\Delta \rho=\Delta \mathbf{z}=\Delta$ [21], [34],

$$
\begin{aligned}
& V(\rho, z)= p_{\rho+} V(\rho+\Delta, z)+p_{\rho-} V(\rho-\Delta, z) \\
&+ p_{z+} V(\rho, z+\Delta)+p_{z_{-}} V(\rho, z-\Delta) \\
& \text { where } \quad p_{z_{+}}=p_{z_{-}}=\frac{1}{4} \\
& p_{\rho_{+}}=\frac{1}{4}+\frac{1}{8 i} \\
& p_{\rho_{-}}=\frac{1}{4}-\frac{1}{8 i}
\end{aligned}
$$

At $\rho=0$, the finite difference equation (23) becomes [21], [34],

$$
\begin{aligned}
V(0, z)= & p_{\rho+} V(\Delta, z)+p_{z+} V(0, z+\Delta) \\
& +p_{z_{-}} V(0, z-\Delta)
\end{aligned}
$$

$$
\text { where } \begin{aligned}
p_{\rho+} & =\frac{4}{6} \\
p_{\rho-} & =0 \\
p_{z+} & =p_{z-}=\frac{1}{6}
\end{aligned}
$$

The transition probabilities of the random walking particles at the interface of the two media $\varepsilon_{1}$ and $\varepsilon_{2}$, assuming $Z=$ constant interface, are determined from Equations (1) - (3). In the section 6 of this paper, simulations are presented for inhomogeneous axisymmetric problems. Two cases are considered based on the choice of the boundary conditions and the prescribed potentials.

\section{Markov Chain Monte Carlo}

A Markov chain is a sequence of random variables $X^{(0)}, X(1), \ldots, X(n)$, where the probability distribution of $X^{(n)}$ is determined by the probability distribution $X^{(n-1)}$
[34]-[38]. The process is random, memoryless, evolving in time and thus, remembers only the most recent past. The conditional probability distributions of the Markov chains are time invariant. Markov chains are mathematical models represented by this kind of process. The Markov chains of interest are discrete-state and discrete-time Markov chains. In the present work, the Markov chain is the random walk, and the states are the grid nodes. The transition probability $P_{i j}$ is the probability that a random-walking particle at node $i$ moves to node $j$ and it is expressed by,

$$
\begin{aligned}
& P_{i j}=P\left(x_{n+1}=j \mid x_{0}, x_{1}, \ldots, x_{n}\right)= \\
& P\left(x_{n+1}=j \mid x_{n}\right), \quad j \in X, n=0,1,2, \ldots
\end{aligned}
$$

The transition probabilities which correspond to the random walks of the random walking particles and form the cornerstone of the MCMC method are derived from the finite difference equations.

The Markov chain is characterized by its transition probability $\mathbf{P}$ defined by

$$
\mathrm{P}=\left[\begin{array}{cccc}
\mathrm{P}_{00} & \mathrm{P}_{01} & \mathrm{P}_{02} & \ldots \\
\mathrm{P}_{10} & \mathrm{P}_{11} & \mathrm{P}_{12} & \ldots \\
\mathrm{P}_{20} & \mathrm{P}_{21} & \mathrm{P}_{22} & \ldots \\
\cdots & \ldots & \ldots & \ldots
\end{array}\right]
$$

$\mathbf{P}$ is a stochastic matrix given by,

$$
\sum_{j \in X} P_{i j}=1, \quad i \in X
$$

Suppose there are $n_{f}$ free (or non-absorbing) nodes and $n_{p}$ fixed (absorbing) nodes, the size of the transition matrix $\mathbf{P}$ is $\mathrm{n}$, where

$$
n=n_{f}+n_{p}
$$

If the absorbing nodes are numbered first and the nonabsorbing states are numbered last, the $n \times n$ transition matrix becomes

$$
\mathbf{P}=\left[\begin{array}{ll}
\mathbf{I} & \mathbf{0} \\
\mathbf{R} & \mathbf{Q}
\end{array}\right]
$$

where

$n_{f} \times n_{p}$ matrix $\mathbf{R}$ represents the probabilities of moving from non-absorbing nodes to absorbing ones;

$n_{f} \times n_{p}$ matrix $\mathbf{Q}$ represents the probabilities of moving from one non-absorbing node to another;

$I$ is the identity matrix representing transitions between the absorbing nodes $\left(P_{i i}=1\right.$ and $\left.P_{i j}=0\right)$;

0 is the null matrix showing that there are no transitions from absorbing to non-absorbing nodes. 
From equation 11, Matrix $\mathbf{Q}$ for axisymmetric region is summarily given as

$Q_{i j}=\left\{\begin{array}{l}\frac{1}{4}, \quad \text { if } i \text { is directly connected to } j \\ \left(\frac{2 i+1}{8 i}\right), \quad \text { if } i \text { is directly connected to } i+1 \\ \left(\frac{2 i-1}{8 i}\right), \quad \text { if } i \text { is directly connected to } i-1 \\ 0, \quad \text { if } i=j \text { or } i \text { is not directly connected to } j\end{array}\right.$

Similarly, from equation 13 , matrix $\mathbf{Q}$, at the line of Symmetry, is summarily defined as

$$
Q_{i j}= \begin{cases}\frac{1}{6}, & \text { if } i=0 \text { and is directly connected } \\ & \text { to } j \text { and } j-1 \\ \frac{4}{6}, & \text { if } i=0 \text { and is directly connected } \\ & \text { to } i+1 \\ 0, & \text { if } i=j \text { or } i \text { is not directly connected } \\ & \text { to } j\end{cases}
$$

The same applies to $R_{i j}$ except that $j$ is an absorbing node. For any absorbing Markov chain, $\mathbf{I}-\mathbf{Q}$ has an inverse called the fundamental matrix

$$
\mathbf{N}=(\mathbf{I}-\mathbf{Q})^{-1}
$$

where $N_{i j}$ is the average number of times the randomwalking particle starting from node $i$ passes through node $j$ before being absorbed. The absorption probability matrix $\mathbf{B}$ is

$$
\mathbf{B}=\mathbf{N} \mathbf{R}
$$

where $\boldsymbol{R}_{i j}$ is the probability that a random-walking particle originating from a non-absorbing node $i$ will end up at the absorbing node $j$. B is an $n_{f} \times n_{p}$ matrix and is stochastic, similar to the transition probability matrix,

$$
\sum_{j=1}^{n_{p}} B_{i j}=1, \quad i=1,2, \ldots, n_{f}
$$

If $\mathbf{V}_{f}$ and $\mathbf{V}_{p}$ contain potentials at the free and fixed nodes, respectively, then

$$
\mathbf{V}_{f}=\mathbf{B} \mathbf{V}_{p}
$$

In terms of the prescribed potentials $V_{1}, V_{2}, \ldots, V_{n p}$, used in Equation (23) becomes

$$
V_{i}=\sum_{j=1}^{n_{p}} B_{i j} V_{j}, i=1,2, \ldots, n_{f}
$$

where $V_{i}$ is the potential at any free node $i$. Unlike Equation (23) and Equation (24) that provide solution at all the free nodes at once, an alternative way to obtain the solution in Equation (24) is to exploit a property of the transition probability matrix $\mathbf{P}$. When $\mathbf{P}$ is multiplied repeatedly by itself a large number of times, we obtain

$$
\begin{aligned}
& \lim _{n \rightarrow \infty} \mathbf{P}^{n}=\left[\begin{array}{ll}
\mathbf{I} & \mathbf{0} \\
\mathbf{B} & \mathbf{0}
\end{array}\right] \\
& \text { Thus, }\left[\begin{array}{c}
\mathbf{V}_{\boldsymbol{p}} \\
\mathbf{V}_{\boldsymbol{f}}
\end{array}\right]=\mathbf{P}^{\boldsymbol{n}}\left[\begin{array}{c}
\mathbf{V}_{\boldsymbol{p}} \\
\mathbf{V}_{\boldsymbol{f}}
\end{array}\right]=\left[\begin{array}{ll}
\mathbf{I} & \mathbf{0} \\
\mathbf{B} & \mathbf{0}
\end{array}\right]\left[\begin{array}{c}
\mathbf{V}_{\boldsymbol{p}} \\
\mathbf{V}_{f}
\end{array}\right]
\end{aligned}
$$

Though Equation (26) can be used to find $V_{f}$ but Equation (23) is more efficient and accurate. However, if $\mathbf{N}$ is accurately calculated in Equation (23) or Equation (26), the solution should be "exact."

\section{Simulation Results}

In this section, simulation results for inhomogeneous axisymmetric problems with MCMC methods are presented for two cases. These include inhomogeneous axisymmetric problem with constant boundary potentials and that with mixed inhomogeneous boundary potentials. Simulation results are reported for both cases.

Table 1: Parameters for Case I.

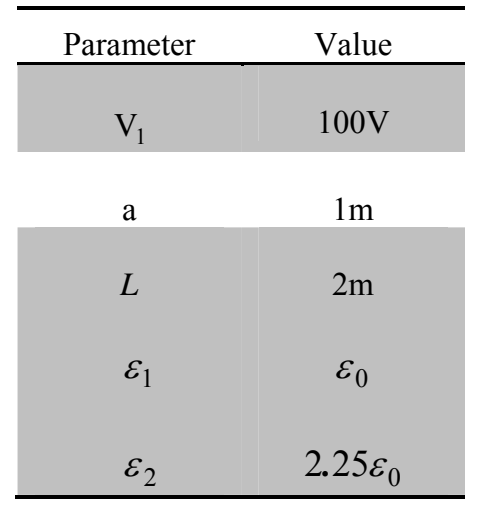


Case I: Axisymmetric Inhomogeneous Problem with Constant Boundary Potential

In this case, axisymmetric inhomogeneous problem given in the Figure 3 is considered with constant boundary potentials. The voltage $V_{1}$ is as given in the Table 1 while $V_{0}$ and $V_{2}$ are grounded respectively. The permittivity for medium 1 and medium 2 are given in the Table 1. With the MCMC procedure given below, simulations are carried out on MATLAB and the results are presented in the Figures 4 and 5. The results for the potential distributions along $\rho=0.5 m, 0 \leq \mathrm{z} \leq 2 \mathrm{~m}, \rho=0,0 \leq \mathrm{z} \leq 2 \mathrm{~m}$ (symmetry line) and $\rho=0.9 m, 0 \leq z \leq 2 \boldsymbol{m}$ are reported in the Figure 4 . In the Figure 5 , the potential distribution along $\mathbf{z}=1,0 \leq \rho \leq 1 \boldsymbol{m}$ (media interface) is reported. The surface plot and the contour plot for all the grid nodes are also presented in the Figure 5. In the Table 2, the MCMC results for selected grid points are compared with the finite difference solution and they are very close.

MCMC Procedure

1. Determine the domain from the given dimensions

2. ... the $\boldsymbol{n}_{f} \times \boldsymbol{n}_{f}$ matrix $\boldsymbol{Q}$ based on the Equation (18) - (19)

3. ... interface transition probabilities from Equation (1)-(3)

4. ... the $\pi_{f} \times \pi_{p}$ matrix $\boldsymbol{R}$ based on the Equation (18)-(19)

5. Compute the fundamental matrix $\mathbf{N}$ from Equation (20)

6. Determine the $\pi_{f} \times n_{p}$ matrix $\mathbf{B}$ on the Equation (21)

7. Compute the prescribed potentials, $V_{1}, V_{2}, \ldots, V_{\text {II }}$.

8. Compute the free node potentials, $V_{f}=B V_{p}$.

9. Plot the results

Table 2: Results Comparison between FDM and MCMC for Axisymmetric Inhomogeneous Problem with Constant Boundary Potential.

\begin{tabular}{ccc}
\hline$(\rho, \mathbf{z})$ & FDM & MCMC \\
\hline$(5,6)$ & 1.1005 & 1.1409 \\
$(7,30)$ & 36.5980 & 36.6260 \\
$(10,21)$ & 7.5901 & 7.5801 \\
$(12,32)$ & 36.1631 & 36.1738 \\
$(16,12)$ & 0.8485 & 0.8618 \\
\hline
\end{tabular}

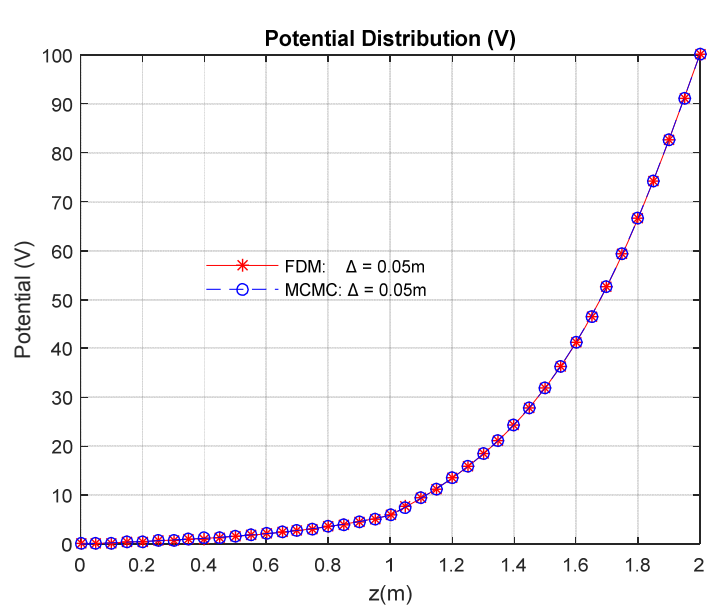

(a)

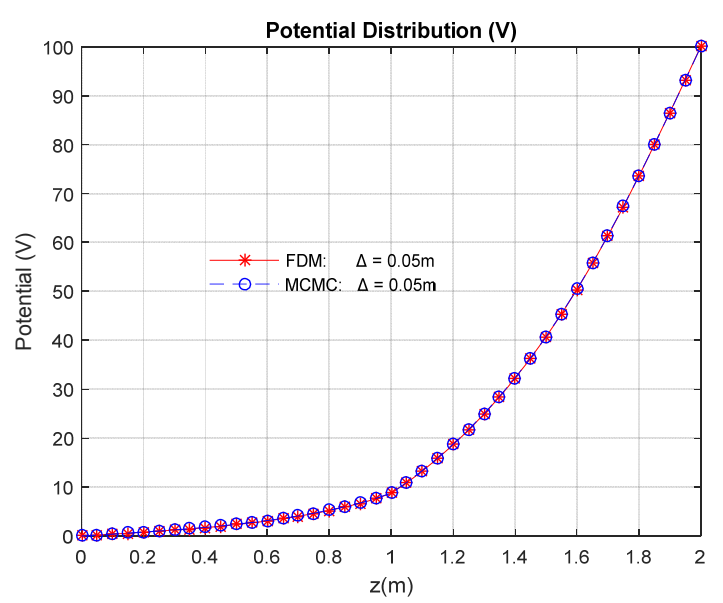

(b)

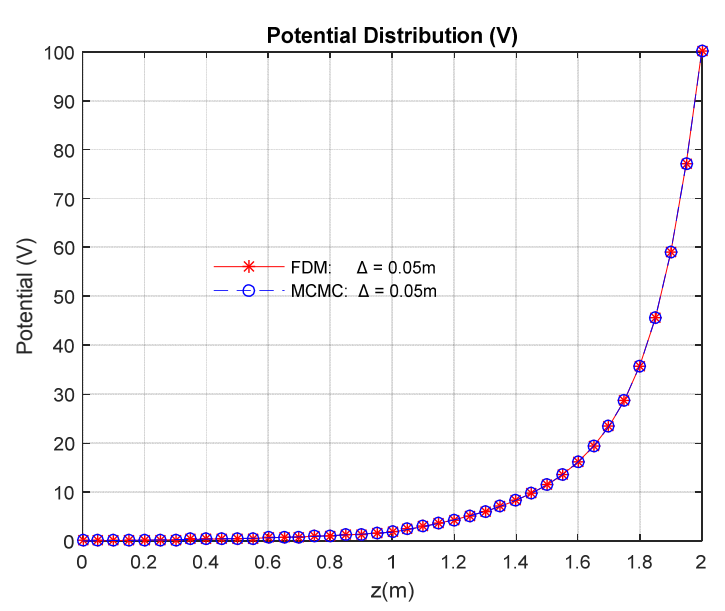

(c)

Figure 4. Potential Distribution along

(a) $\rho=0.5 \mathrm{~m}, 0 \leq \mathrm{z} \leq 2 \mathrm{~m}$ (b) $\rho=0,0 \leq \mathrm{z} \leq 2 \mathrm{~m}$ (line of symmetry) (c) $\rho=0.9 \mathrm{~m}, 0 \leq \mathrm{z} \leq 2 \mathrm{~m}$ for Axisymmetric Inhomogeneous Problem with constant boundary potentials. 


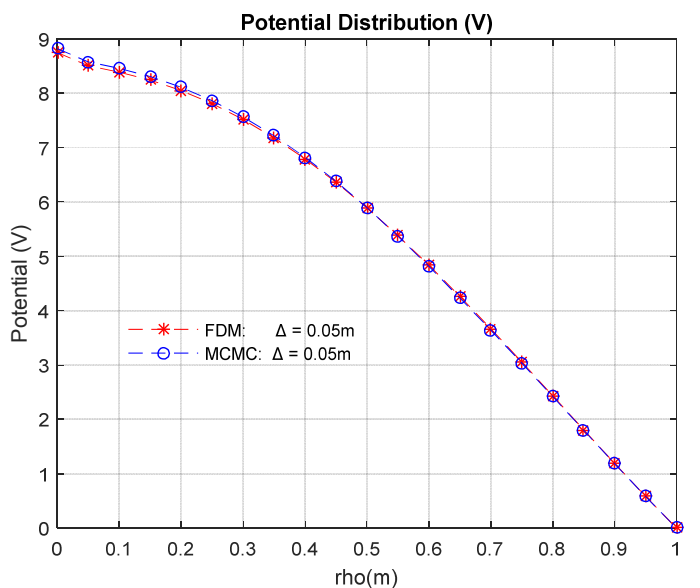

(a)

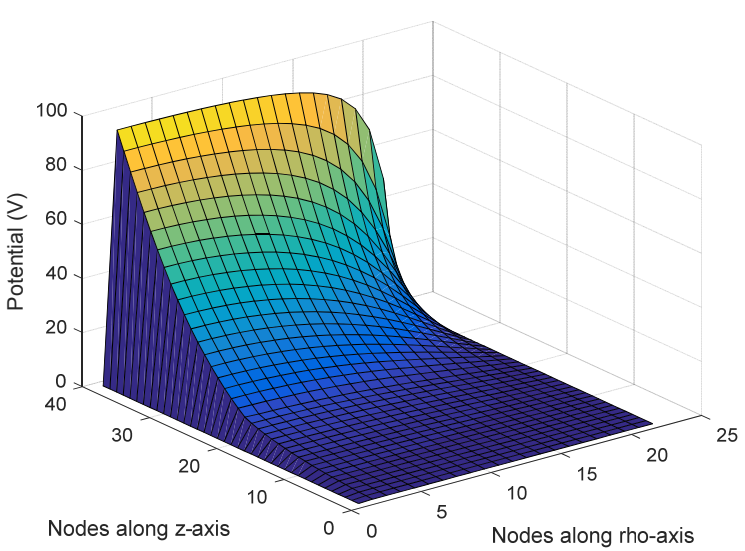

(b)

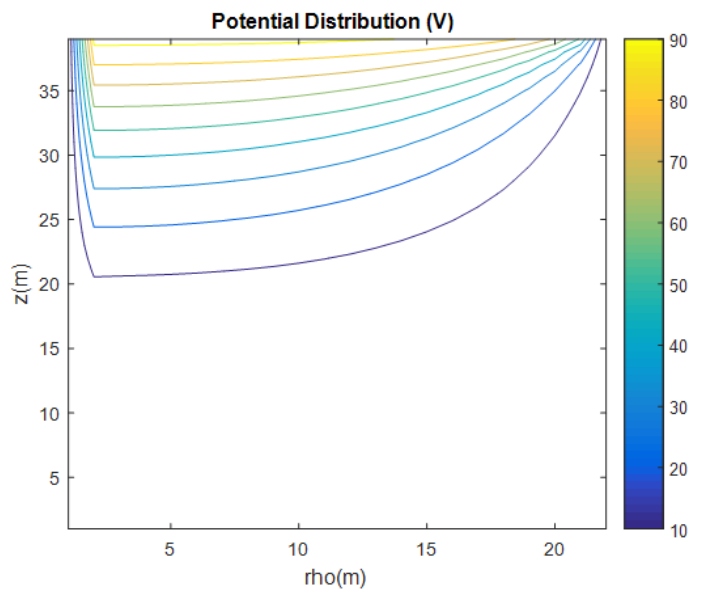

(c)

Figure 5. Potential Distribution along (a) $\mathrm{z}=1,0 \leq \rho \leq 1 \mathrm{~m}$ (media interface), (b) Surface Plot (c) Contour Plot for Axisymmetric Inhomogeneous Problem with constant boundary potentials.
Table 3: Parameters for Case II.

\begin{tabular}{cc}
\hline Parameter & Value \\
\hline $\mathrm{V}_{1}$ & $50 \mathrm{~V}$ \\
$\mathrm{a}$ & $1 \mathrm{~m}$ \\
$L$ & $2 \mathrm{~m}$ \\
$\varepsilon_{1}$ & $\varepsilon_{0}$ \\
$\varepsilon_{2}$ & $2.25 \varepsilon_{0}$ \\
\hline
\end{tabular}

Case II: Axisymmetric Inhomogeneous Problem with Mixed Boundary Potentials

Suppose the axisymmetric problem given in the Figure (3) has its ends $\boldsymbol{z}=0$ and $\boldsymbol{z}=\boldsymbol{L}$ held at zero potential and $50 \mathrm{~V}$ respectively. If voltage, $\mathrm{V}(\mathrm{a}, \mathrm{z})$ is given as [34]:

$$
\mathrm{V}(\mathrm{a}, \mathrm{z})= \begin{cases}\mathrm{V}_{0} \frac{\mathrm{z}}{\mathrm{L}}, & 0<\mathrm{z}<\frac{\mathrm{L}}{2} \\ \mathrm{~V}_{0}\left(1-\frac{\mathrm{z}}{\mathrm{L}}\right), & \frac{\mathrm{L}}{2}<\mathrm{z}<\mathrm{L}\end{cases}
$$

In this section, axisymmetric inhomogeneous problem with constant and inhomogeneous potentials is presented. The voltage $\mathrm{V}_{0}$, is defined as in the equation (28), $\mathrm{V}_{1}$ is fixed at $50 \mathrm{~V}$ as given in the Table 3 and the cylinder is grounded at voltage $\mathrm{V}_{2}$. The permittivity for medium 1 and the medium 2 are given respectively as in the Table 3. The MCMC procedures used for case I are repeated and the simulations are carried out on MATLAB. The simulation results are reported in the Figures 6 . The potential distribution along $\rho=0.5 m, 0 \leq z \leq 2 m, \rho=0,0 \leq z \leq 2 m$ (symmetry line), $\rho=0.9 \boldsymbol{m}, 0 \leq \mathbf{z} \leq 2 \boldsymbol{m}, \mathbf{z}=1,0 \leq \rho \leq 1 \boldsymbol{m}$ (media interface), surface plot and the contour plot for all the grid nodes are reported in the Figure 6. In the Table 4, the MCMC results for selected grid points are compared with the finite difference solution. The results are very close.

Table 4: Results Comparison between FDM and MCMC for Problems with Mixed Boundary Potentials.

\begin{tabular}{ccc}
\hline$(\rho, \mathbf{z})$ & FDM & MCMC \\
\hline$(5,6)$ & 11.2701 & 11.3465 \\
$(7,30)$ & 35.8286 & 35.7711 \\
$(10,21)$ & 31.5857 & 31.2791 \\
$(12,32)$ & 34.2649 & 34.2102 \\
$(16,12)$ & 26.7636 & 26.7633 \\
\hline
\end{tabular}




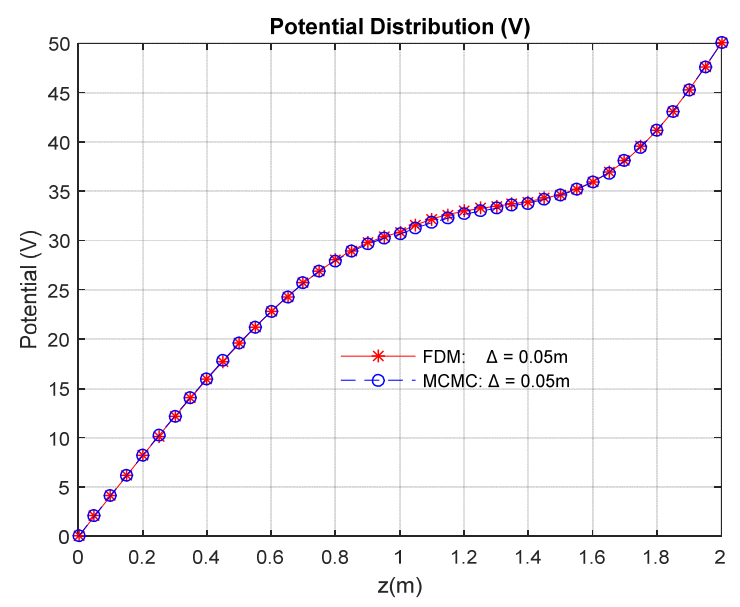

(a)

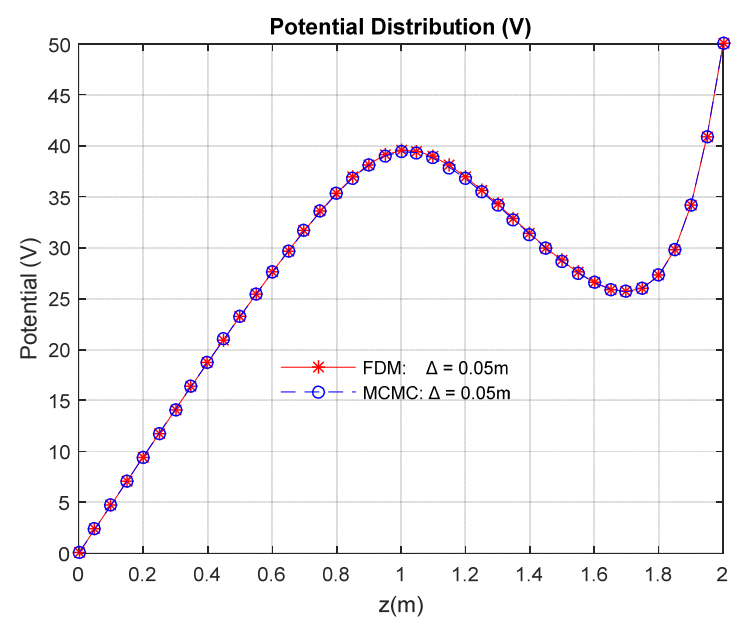

(c)

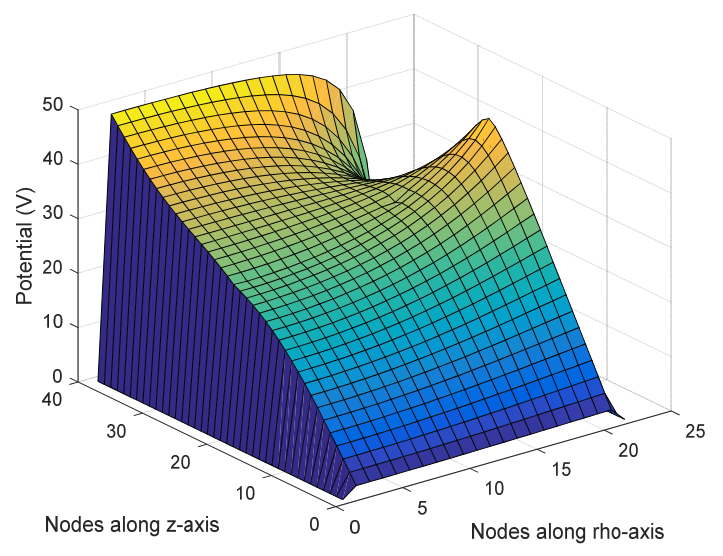

(e)

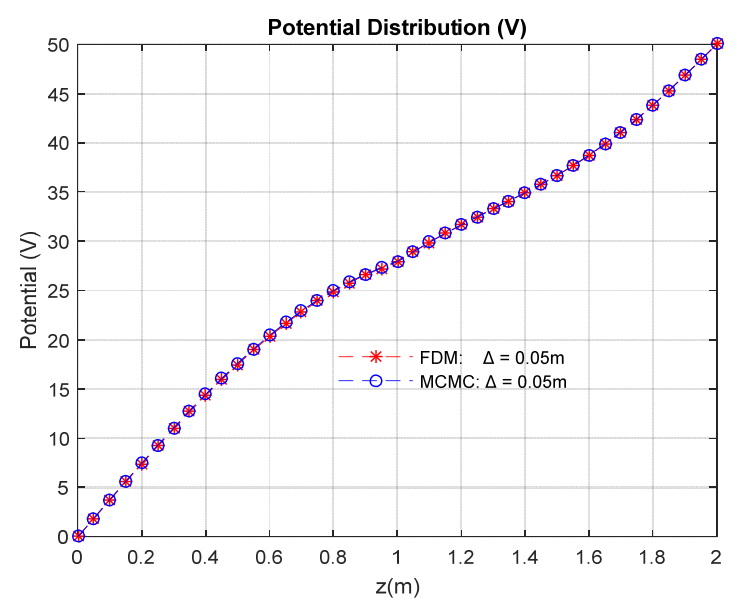

(b)

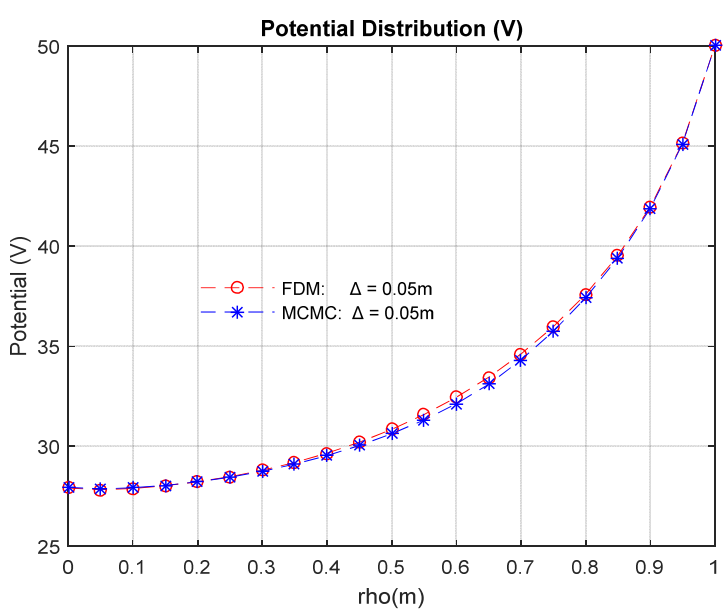

(d)

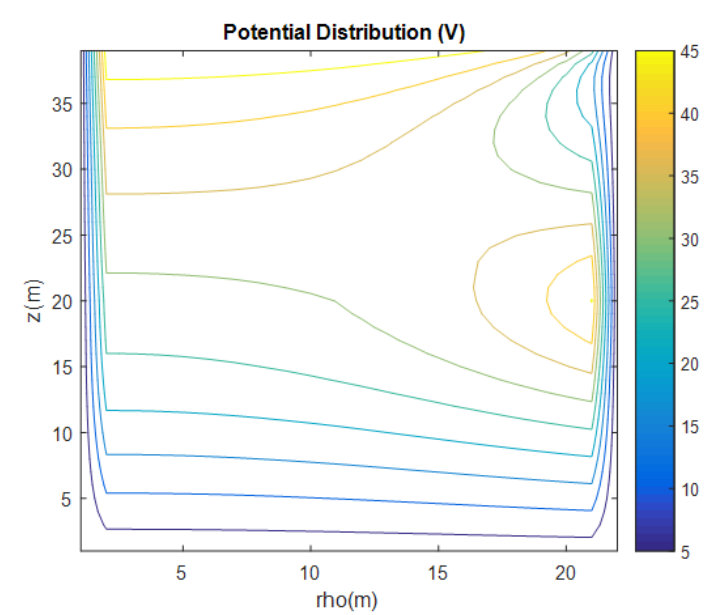

(f)

Figure 6. Potential Distribution along (a) $\rho=0.5 \mathrm{~m}, 0 \leq \mathrm{z} \leq 2 \mathrm{~m}$; (b) $\rho=0,0 \leq \mathrm{z} \leq 2 \mathrm{~m}$ (line of symmetry) (c) $\rho=0.9 \mathrm{~m}, 0 \leq \mathrm{z} \leq 2 \mathrm{~m}$ (d) $\mathrm{z}=1,0 \leq \rho \leq 1 \mathrm{~m}$ (media interface), (e) Surface Plot (f) Contour Plot for Axisymmetric Inhomogeneous Problem with mixed boundary potentials. 


\section{Conclusion}

With growing cost of data storage and increasing complexity of electromagnetic problems facing device designers today, opportunity frequently exists to approximate problem domains to reduce problem sizes and resultant data storage requirements during implementation. Most numerical methods available nowadays involve huge iterative procedures often with large data storage requirements. The Markov Chain Monte Carlo (MCMC) solutions presented in this paper is fast and efficient in implementation once matrices $\boldsymbol{Q}$ and $\boldsymbol{R}$ are accurately calculated. In the paper, inhomogeneous axisymmetric problems are considered. Specifically, Laplace's equations in inhomogeneous cylindrical system are solved using axisymmetric approximations. Two cases of the problem based on constant and mixed boundary potentials are considered. The MCMC solutions presented in this paper agree well with the finite difference method while using less computational resources as the method requires no iterations.

The MCMC method presented in this paper can be extended to homogeneous and inhomogeneous axisymmetric Poisson's equations as well as axisymmetric problems in spherical coordinates.

\section{References}

[1] V. F. Fusco and P. A. Linden, "A Markov Chain Approach for Static Field Analysis," Microwave and Optical Technology Letters, vol. 1, no. 6, 1988, pp. 216220.

[2] A. E. Shadare, M. N. O. Sadiku and S. M. Musa, "Analysis of Microstrip Line using Markov Chain Monte Carlo," International Conference on Scientific Computing, 2015, pp. 135-139.

[3] A. E. Shadare, M. N. O. Sadiku and S. M. Musa, "Solution to Poisson's Equation in Rectangular Inhomogeneous Dielectric Media with the Markov Chain Monte Carlo," International Conference on Scientific Computing, 2017, pp. 10-15.

[4] M. N. O. Sadiku, R. C. Garcia, S. M. Musa and S. R. Nelatury, "Markov Chain Monte Carlo Solution of Poisson's Equation," International Journal on Recent and Innovative Trends in Computing and Communication, vol. 3, Issue 1, January 2015.

[5] K. Gu and M. N. O. Sadiku, "Absorbing Markov Chain Solution for Poisson's Equation," Proceedings of the IEEE Southeastcon, 2000, pp. 297-300.

[6] R. C. Garcia, M. N. O. Sadiku and K. Gu, "Applying Absorbing Markov Chains to Solve Poisson's Equation in Inhomogeneous Regions," Proceedings of the IEEE Southeastcon, 2001, pp. 166-168.

[7] P. R. P. Hoole and A. J. Pearmain, "A Review of the Finite-Difference Method for Multidielectric Electrostatic Field problems with Sharp-Edged
Electrodes," Electric Power Systems Research, 24 (1992), pp. 19-30.

[8] F. Sanchez-Quesada et al., "Monte-Carlo Method for Discrete Inhomogeneous Problems," Proceedings of the Institution of Electrical Engineers, 1978, vol. 125, Issue 12, pp. 1400-1402.

[9] R. Schlott, "A Monte Carlo Method for the Dirichlet Problem of Dielectric Wedges," IEEE Transactions on Microwave Theory and Techniques, 1988, vol. 36, no. 4, pp. 724-730.

[10] R. C. Garcia and M. N. O. Sadiku, "Monte Carlo FixedRadius Floating Random Walk Solution for Potential Problems," Proceedings of the IEEE Southeastcon, 1996, pp. 88-91.

[11] J. H. Pickles, "Monte-Carlo Calculation of Electrically Induced Human-Body Currents" IEE Science Proceedings, Vol. 134, Pt. A, No. 9, pp. 705-711, November 1987.

[12] M. N. O. Sadiku and R. C. Garcia, "Monte Carlo Floating Random Walk Solution of Poisson's Equation," Proceedings of Southeastcon, 1993.

[13] K. Gu and M. N. O. Sadiku, "A triangular mesh random walk method for Dirichlet Problem," J. Franklin Inst., March, 1995, pp. 569-578.

[14]M. N. O. Sadiku, S. O. Ajose, and Zhibao Fu, "Applying the Exodus Method to Solve Poisson's Equation," IEEE Trans. Microwave Theory and Techniques, vol. 42, no.4, April, 1994, pp. 661-666.

[15] N. J. Jayant and L. L. C. Yannick, "An Improved Floating-Random-Walk Algorithm for Solving the Multi-Dielectric Dirichlet Problem," IEEE Transactions on Microwave Theory and Techniques, 1993, vol. 41, no. 2, pp. 325-329.

[16] G. E. Zinsmeiter and J. A. Sawyerr, "A method for improving the efficiency of Monte Carlo Calculation of heat conduction problems," Transactions of the ASME, Journal of Heat Transfer, vol. 96, 1974, pp. 246-248.

[17] G. E. Zinsmeiter and S. S. Pan, "A Modification of the Monte Carlo Method," International Journal for Numerical Methods in Engineering, vol. 10, 1976, pp. 1057-1064.

[18] V. F. Fusco and P. A. Linden, "A Markov Chain Approach for Static Field Analysis," Microwave and Optical Technology Letter, vol. 1, no. 6, August 1988, pp. 216-220.

[19] M. N. O. Sadiku and R. C. Garcia, "Whole Field Computation Using Monte Carlo Method," International Journal of Numerical Modelling: Electronic Networks, Devices and Fields, vol. 10, 1997, pp. 303-312.

[20] M. N. O. Sadiku and K. Gu, "Floating random walk method on axisymmetric potential problems," 1994 International Symposium on Electromagnetic Compatibility, Sendia, Japan, May, 1994, pp. 659-662.

[21] M. N. O. Sadiku, "Monte Carlo Solution of Axisymmetric Potential Problems," IEEE Transactions on Industry Applications, vol. 29, no. 6, 1993, pp. $1042-1046$. 
[22]F. Z. Louai, N. N. Said and S. Drid, "Numerical Analysis of Electromagnetic Axisymmetric Problems Using Element Free Galerkin Method," Journal of Electrical Engineering, vol. 57, No. 2, 2006, pp. 99104.

[23] A. N. Hayati, M. M. Ahmadi, and S. A. Sadrnejad, "Analysis of Axisymmetric Problems By Element-Free Galerkin Method," International Journal of Modeling and Optimization, vol. 2, No. 6, December 2012.

[24] R. D. Soares, F. J. S. Moreira, R. C. Mesquita, D. A. Lowther and N. Z. Lima, "A Modified Meshless Local Petrov-Galerkin Applied to Electromagnetic Axisymmetric Problems," IEEE Transactions on Magnetics, vol. 50, No. 2, February 2014.

[25] J. B. M. Melissen and J. Simkin, "A New Coordinate Transform For The Finite Element Solution of Axisymmetric Problems In Magnetostatics," IEEE Transactions on Magnetics, vol. 26, No. 2, March 1990.

[26]A. Boglietti, M. Chiampi, D. Chiarabaglio and M. Tartaglia, "Finite Element Approximation In Axisymmetrical Domains," IEEE Transactions on Magnetics, vol. 26, No. 2, March 1990

[27]A. Yoneta, M. Tsuchimoto and T. Honma, "An Analysis of Axisymmetric Modified Helmholtz Equation By Using Boundary Element Method," IEEE Transactions on Magnetics, vol. 26, no. 2, March 1990.

[28] M. N. O. Sadiku and Raymond C. Garcia, "Method of Lines Solution of Axisymmetric Problems," Proceedings of the IEEE Southeastcon, 2000, pp. 527530.

[29] S. Gratkowski, T. Todaka, M. Enokizono, and R. Sikora, "Asymptotic Boundary Conditions for the Finite Element Modeling of Axisymmetric Electrical Field Problems," IEEE Transactions on Magnetics, vol. 36, No. 4, pp. 717-721, July 2000.
[30]Q. Chen, A. Konrad and S.Baronijan, "Asymptotic Boundary Conditions for Axisymmetric Finite Element Electrostatic Analysis,' " IEEE Transactions on Magnetics, vol. 30, No. 6, pp. 4335-4337, November 1994.

[31]M. Ehrich, J. Kuhlmann and D. Netzler, "High Accuracy Integration of Boundary Integral Equations Describing Axisymmetric Field Problems," Asia Pacific Microwave Conference, 1997, pp. 462-464.

[32] L. Krahenbuhl and A. Nicolas, "Axisymmetric Formulation for Boundary Integral Equation Methods in Scalar Potential Problems," IEEE Transactions on Magnetics, vol. MAG-19, NO. 6, pp. 2364- 2366, November 1983.

[33] E. Godoy, V. Boccardo and M. Durán, “A Dirichlet-toNeumann Finite Element Method for Axisymmetric Elastostatics in a Semi-Infinite Domain," Journal of Computational Physics, 2017, pp. 1-26.

[34] M. N. O. Sakidu, Computational Electromagnetics with MATLAB. Boca Raton, FI, Boca Raton, CRC Press, Fourth Edition, 2019

[35]M. N. O. Sakidu, Monte Carlo Methods for Electromagnetics. CRC Press, Boca Raton, FI, 2009.

[36] M. N. O. Sadiku, "Monte Carlo Methods in an Introductory Electromagnetic Course," IEEE Transactions on Education, 33, 1, February 1990, pp. 73-80.

[37] M. A. Kolbehdari and M. N. O. Sadiku, "Finite \& Infinite Element Analysis of Coupled Cylindrical Microstrip Line in a Nonhomogeneous Dielectric Media," Proceedings of IEEE Southeastcon, 1995. pp. 269-273.

[38] M. N. O. Sadiku and D. T. Hunt, "Solution of Dirichlet Problems by the Exodus Method," IEEE Transactions on Microwave Theory and Techniques, 40, 1, January 1992, pp. 89-95. 